\title{
Deshidratación de plátano (Musa paradisiaca) por medio de radiación solar en un secador directo
}

\author{
Dehydration of banana (Musa paradisiaca) by means of solar radiation in a direct \\ dryer
}

CARRILLO-CARRILLO, Manuela $\dagger^{*}$, CASTORENA-ALEMÁN, Juan Daniel, GARCÍA-JIMENÉZ, Fátima de los Ángeles y GARCÍA-GONZÁLEZ, Juan Manuel

Universidad Autónoma de Zacatecas

ID $1^{\text {er }}$ Autor: Manuela, Carrillo-Carrillo / ORC ID: 0000-0002-6196-0561

ID $1^{\text {er }}$ Coautor: Juan Daniel, Castorena-Alemán / ORC ID: 0000-0002-7925-8279

ID $2{ }^{\mathrm{do}}$ Coautor: Fátima de los Ángeles, García-Jimenéz / ORC ID: 0000-0002-2755-5458

ID $3^{\text {er }}$ Coautor: Juan Manuel, García-González / ORC ID: 0000-0001-7259-5021, CVU CONACYT ID: 346241

\section{Resumen}

El objetivo es deshidratar plátano (Musa paradisiaca) empleando dos secadores solares directos. La finalidad de deshidratar el plátano, es darle un valor agregado. La fruta se descáscara, y se corta en rodajas de $1.75 \mathrm{~cm}$ de radio con un espesor de $0.525 \mathrm{~cm}$. Se coloca en dos charolas de malla polimérica con dimensiones de $24 \mathrm{~cm}$ por $34.2 \mathrm{~cm}$. Se le determina la humedad inicial, y se pesan. Posteriormente se introducen a los secadores solares. Para ambos secadores se determina la humedad y la temperatura del medio cada 15 min, y cada hora se determina el color. Por último, se determina el porcentaje de humedad del producto final. Cada prueba realizada se hace en triplicado. El porcentaje de humedad disminuye desde un $57.23 \%$ hasta un 20.87 $\%$ en convección natural y $20.80 \%$ en convección forzada. El tiempo de secado es de 8 horas y la temperatura promedio de operación es de $38.7^{\circ} \mathrm{C}$ para la convección natural y $34.68{ }^{\circ} \mathrm{C}$ para la convección forzada. En la deshidratación el porcentaje de humedad disminuye un $36.36 \%$ en promedio en las dos formas de operación. En convección forzada el producto se obscurece menos.

\begin{abstract}
The objective is to dehydrate banana (Musa paradisiaca) using two direct solar dryers. The purpose of dehydrating the banana, is to give an added value. The fruit is remove the peel, and cut into slices of 1.75 $\mathrm{cm}$ radius with a thickness of $0.525 \mathrm{~cm}$. It is placed in two polymer mesh trays with dimensions of $24 \mathrm{~cm}$ by $34.2 \mathrm{~cm}$. The initial moisture is determined, and weighed. Later they are introduced to solar dryers. For both dryers the humidity and the temperature of the medium are determined every $15 \mathrm{~min}$, and the color is determined every hour. Finally, the moisture content of the final product is determined. Each test is done in triplicate. The percentage of humidity decreases from $57.23 \%$ to $20.87 \%$ in natural convection and $20.80 \%$ in forced convection. The drying time is 8 hours and the average operating temperature is $38.7^{\circ} \mathrm{C}$ for natural convection and $34.68^{\circ} \mathrm{C}$ for forced convection. In dehydration the percentage of humidity decreases $36.36 \%$ on average in the two forms of operation. In forced convection the product darkens less.
\end{abstract}

Dehydrated, Banana, Dried

Citación: CARRILLO-CARRILLO, Manuela, CASTORENA-ALEMÁN, Juan Daniel, GARCÍA-JIMENÉZ, Fátima de los Ángeles y GARCÍA-GONZÁLEZ, Juan Manuel. Deshidratación de plátano (Musa paradisiaca) por medio de radiación solar en un secador directo. Revista de Sistemas Experimentales. 2019. 6-19: 19-23

\footnotetext{
* Correspondencia al Autor (Correo electrónico: jmgarcia@uaz.edu.mx)

$\dagger$ Investigador contribuyendo como primer Autor
} 


\section{Introducción}

Las frutas son el grupo de alimentos indispensables para la salud y el bienestar humano. Su aporte en fibra, vitaminas, minerales y antioxidantes, son necesarios para la vida del hombre. Desde años atrás se ha observado el interés sobre los beneficios de los productos vegetales en la reducción de diferentes enfermedades, el problema se presenta cuando son productos con una estacionalidad marcada y con muy alto contenido en agua. Además, los hábitos de las personas han cambiado con el tiempo, actualmente se nota ante la demanda de alimentos que sean duraderos. Por esta parte es importante por el ato contenido de agua con el cual cuenta la mayoría de las frutas, se hace necesaria la deshidratación para reducir el ataque microbiano.

Actualmente la fruta deshidratada se ha comercializado como un postre y en la repostería. Se emplea en la elaboración de alimentos, por ejemplo para deportistas. El secado o deshidratación es una técnica para conservar los alimentos donde el objetivo principal es retirar la mayor parte de agua de los mismos donde a partir de un flujo de aire calentado con radiación solar pasa a través del alimento. Durante este proceso puede haber perdida de nutrientes esto dependerá de las condiciones que se presenten (humedad del medio ambiente, temperatura, velocidad del viento, etc.). Existen varias formas en la deshidratación de alimentos como lo son la forma natural al sol (convección natural) o de forma mecánica (convección forzada).

El objetivo de este trabajo es la deshidratación del plátano, mediante el uso de secadores solares directos de caseta en operación por convección natural $(\mathrm{CN})$ y convección forzada $(\mathrm{CF})$, con el fin de dar una aplicación de snack, dando un mayor valor agregado y de mayor tiempo de anaquel. El plátano es el cuarto cultivo a nivel mundial después del trigo, arroz y maíz, Nativo del sudeste asiático (entre la India y el este de la península de Malaya), que llegó a América a través del misionero católico Tomás Berlanga a República Dominicana en 1516 (Paiva Purizaca, 2019). Es un fruto comestible de grandes plantas herbáceas del género Musa, tiene cualidades variables en tamaño, color y firmeza, alargado, generalmente curvado y carnoso.
Un árbol de plátano puede contener de 5 a 20 cabezas o piñas que cuelgan de la parte superior de la planta y cada una de esta de 2 a 20 frutos (Dejo, 2018). Es rico en almidón cubierto con una cáscara que puede ser verde, amarilla o marrón dependiendo de su madurez, la cual puede ser empleada para la elaboración de harina para galletas aprovechando su alto contenido en fibra (Dejo, 2018).

El plátano por su contenido de hidratos de carbono proporciona un valor calórico elevado, los nutrientes más representativos del plátano son el potasio, vitamina $\mathrm{C}$, fósforo, el magnesio, el ácido fólico y sustancias de acción astringente; sin despreciar su elevado aporte de fibra, del tipo fruto-oligosacáridos, es rico en dopamina, de efecto vasoconstrictor, y serotonina, que regula la secreción gástrica y estimula la acción intestinal (Dejo, 2018). En la Tabla 1 se presenta los principales nutrientes del plátano, así como su concentración por cada $100 \mathrm{~g}$.

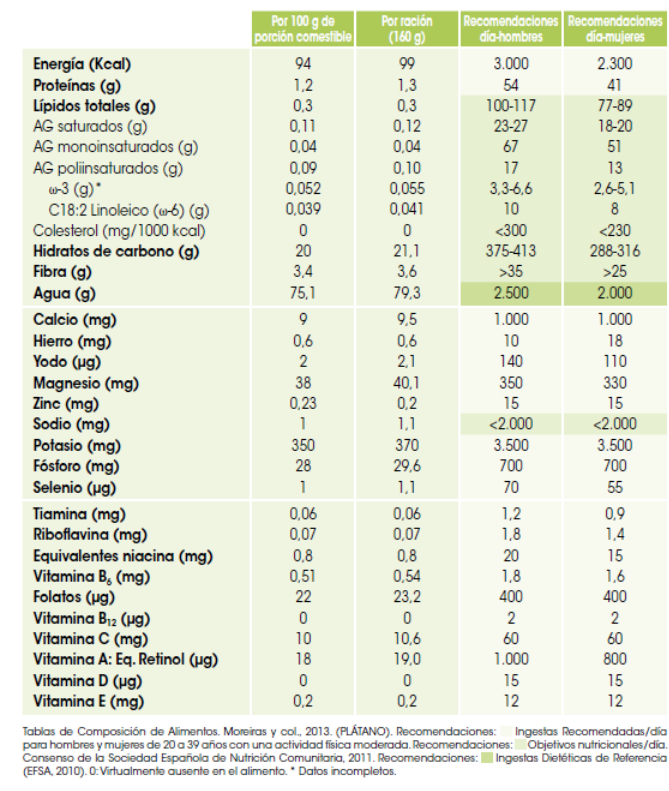

Tabla 1 Valor nutricional del plátano por cada 100g, (Valero, 2018)

Durante el proceso de secado se produce transferencia de calor entre los alrededores y la superficie del plátano, así como, transferencia de masa del agua contenida en el plátano, provocando el paso al transporte de esta al aire empleado en el secado.

Ya que el secado se realiza utilizando la radiación solar, se debe considerar las hora sol y la cantidad de irradiancia presente durante la experimentación. 
Dentro de la literatura se encontraron algunos trabajos similares al estudio realizado en el presente. Carranza et al (2002) usaron un horno secador que operaba en convección forzada para deshidratar y posteriormente obtener harinas de plátano, en su estudio determinaron las condiciones óptimas de secado (Carranza J., 2002). Ángel (2015) evaluó el proceso de secado de pulpa de plátano empleando un deshidratador solar, encontrando que entre más desecado está el fruto la cantidad de azucares del producto se incrementa (Ángel Nolasco, 2015).

Figueredo y González (2016), deshidrataron plátano utilizando secado directo con flujo axial de aire, lo realizaron desde temperatura ambiente hasta $96{ }^{\circ} \mathrm{C}$, alcanzando una humedad final de $6.3 \%$ (Figueredo Rondón M.C., 2016). De la Vega (2017), diseñó y construyó un equipo deshidratador solar, el cual deshidrató hasta un $12.7 \%$ de humedad final en el plátano (De la Vega, 2017). Hernández et al (2017) secaron frutas y verduras con energía solar, reduciendo el costo de producción por los gastos de energía eléctrica (Hernández, 2017).

\section{Materiales y métodos}

Para este trabajo se empleó plátano (Musa paradisiaca) fresco con una maduración similar. Primeramente, se lavaron y se descascaron, luego se cortó en rodajas con un diámetro de $3.50 \pm 0.1 \mathrm{~cm}$ y un espesor de $0.5 \pm 0.025 \mathrm{~cm}$. Se prosiguió a colocar las rodajas en charolas de malla polimérica con dimensiones de $0.24 \mathrm{~m}$ por $0.342 \mathrm{~m}$. Las charolas se enumeraron, se pesaron antes y después de colocar las rodajas de plátano. Una propiedad organoléptica muy importante en el proceso de frutas es su color.

Al momento de que un producto se introduce al mercado las personas se fijan en el aspecto físico, de ahí de que el color es algo importante. Para determinar el cambio en el color se utilizó un colorímetro TPM, se tomaron tres muestras al inicio del proceso para tal fin y posteriormente se determinó cada hora durante el proceso de deshidratación, hasta el término de este. Además, se utilizó el sistema CIElab para realizar la evaluación del color del plátano. Este sistema trabaja en coordenadas tridimensionales, donde $\mathrm{L}$ es el eje, a y b son los ejes horizontales como se muestra en la figura 1.

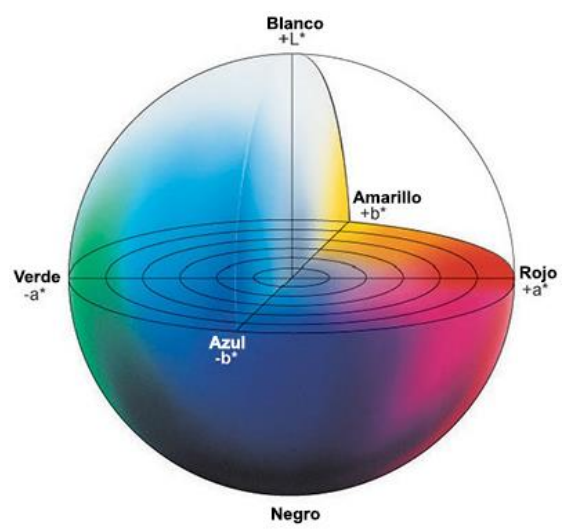

Figura 1 Diagrama del sistema CIElab, (Minolta, 2014)

Los valores que se obtuvieron con el colorímetro, fueron utilizados para el cambio de color total, donde se hizo uso de la Ec. 1, donde $\Delta \mathrm{L}$ es la diferencia de luminosidad, $\Delta \mathrm{a}$ es la diferencia entre rojo-verde y $\Delta b$ la diferencia entre amarillo-azul; el sentido de la desviación se indica con el valor y por el signo de las deltas individuales.

$$
\Delta E=\Delta L^{2}+\Delta a^{2}+\Delta b^{2}
$$

Las charolas con las rodajas de plátano se introdujeron dentro de un secador solar directo de acrílico con dimensiones de $0.74 \mathrm{~m}$ por 0.80 $\mathrm{m}$ de base y una altura frontal de $0.13 \mathrm{~m} \mathrm{y}$ posterior de $0.40 \mathrm{~m}$; el cual cuenta con perforaciones circulares pequeñas para así permitir la entrada y salida del aire. Para la operación en convección forzada, en la parte trasera de la caseta se colocó un ventilador para suministrar el aire necesario en la deshidratación, así como, para desplazar el que ya estaba humidificado.

La parte frontal de la caseta se instaló con una orientación al sur. Durante la primera hora de experimentación se pesan las charolas cada 15 minutos, posteriormente se tasan cada 30 minutos y se registra el peso, de igual manera se registra la temperatura interna de las casetas, empleando dos termómetros Checktemp. Se midió la humedad al inicio y al final de las pruebas con una termobalanza OHAUS MB45. La irradiancia global se determinó mediante un piranámetro CMP 22 de Kipp \& Zone, la irradiancia directa se midió mediante un piraheliometro Eppley, para medir la velocidad y dirección del viento se utilizó un anemómetro y veleta RM-Young, para la temperatura y la humedad en el medio ambiente se empleó un termohigrómetro Vaisala. 
Instrumentos ubicados en la Estación Solarimétrica Zacatecas_04 del Sistema Solarimétrico Mexicano que se encuentra instalada en el edificio 6 perteneciente al Programa de Ingeniería Química en el Campus UAZ Siglo XXI en Zacatecas, Zac.

\section{Resultados y discusión}

Los datos recuperados de la Estación Zacatecas_04 promedio durante la operación de secado del plátano son: La irradiancia solar global fue de $540 \mathrm{~W} / \mathrm{m}^{2}$, la irradiancia solar directa fue de $560 \mathrm{~W} / \mathrm{m}^{2}$, la presión barométrica de 770.2 mbar, la velocidad del viento de 4.25 $\mathrm{m} / \mathrm{s}$ y dirección del viento $200^{\circ} \mathrm{SO}$, la temperatura de $25^{\circ} \mathrm{C}$ y la humedad relativa de $18.3 \%$.

Para la determinación del color se obtuvo en la convección natural (figura 2) el plátano adquirió un color obscuro conforme se incrementó el tiempo en el secador, mientras que en la operación por convección forzada (figura 3) se observó que el plátano mantenía su color natural sin ser afectado, como en la convección natural.
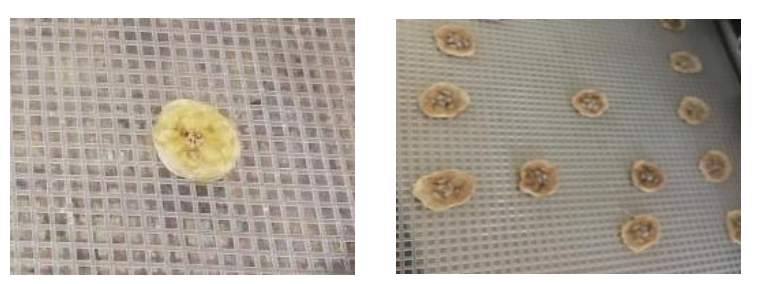

Figura 2 Cambio de color del plátano en la operación por convección natural
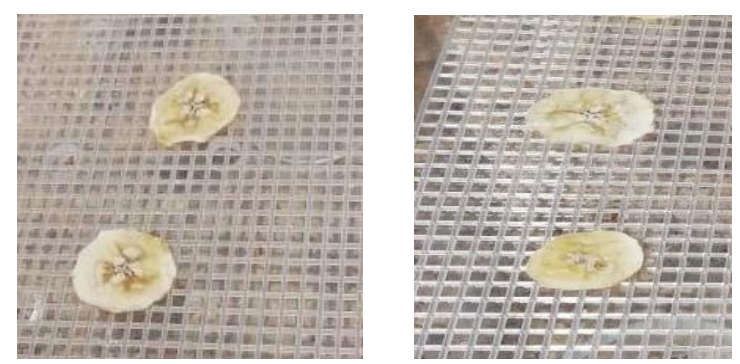

Figura 3 Cambio de color del plátano mediante el proceso de convección forzada

En la tabla 2 se muestran los resultados que se obtuvieron en la deshidratación del plátano en los secadores directos por convección natural y forzada. Para obtener esta comparación de ambos regímenes de operación, se consideró un tiempo de secado de $8 \mathrm{~h}$.

\begin{tabular}{|l|l|l|} 
Convección & $\mathrm{T}_{\text {prom }}\left({ }^{\circ} \mathrm{C}\right)$ & $\% \mathrm{H}_{\text {final }}$ \\
\cline { 2 - 3 } Natural & $38.89 \pm 5.63$ & 20.87 \\
Forzada & $34.11 \pm 3.24$ & 20.80 \\
\hline
\end{tabular}

Tabla 2 Resultados de la deshidratación de plátano empleando convección natural y forzada

En el gráficos 1 se observa como es la pérdida de peso en el tiempo de deshidratación en convección natural y convección forzada. La curva característica de secado que se obtuvo en la convección forzada, fue debido a la influencia del flujo de aire suministrado, ya que, removía y retiraba el aire que se encontraba dentro del secador modificando la temperatura interna del secador. En el gráfico 2 se observa como la radiación solar durante el proceso de secado tiene más influencia en la convección natural ya que alcanza mayor temperatura que la forzada.

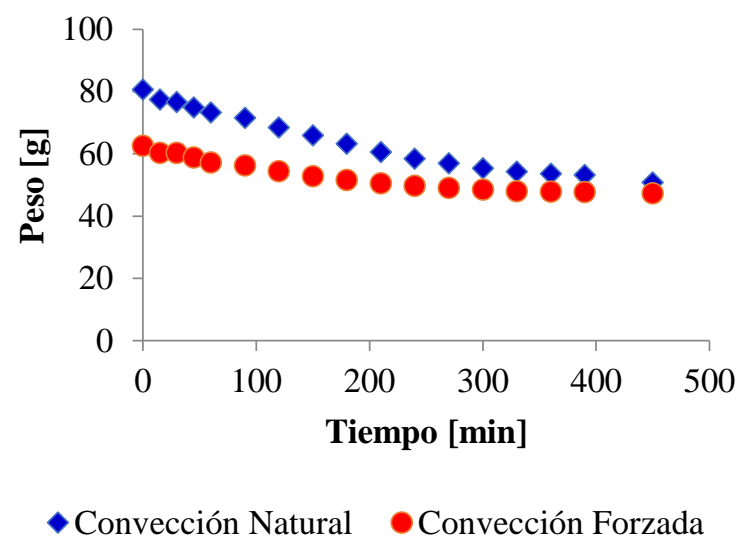

Grafico 1 Pérdida de peso con respecto al tiempo de secado

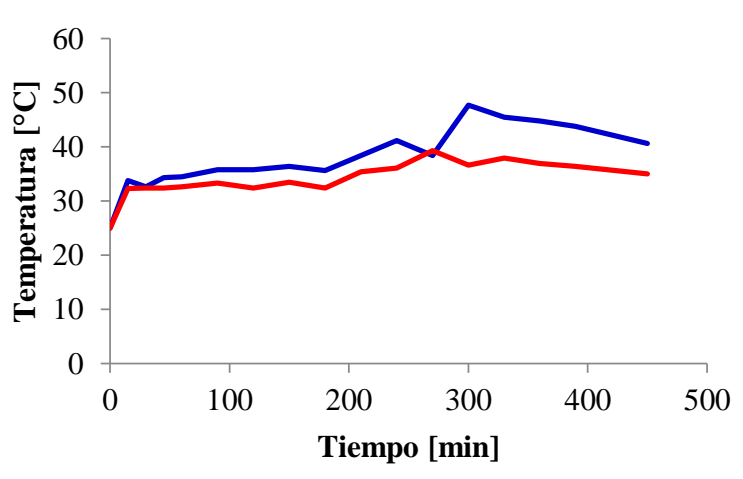

—Convección Natural Convección Forzada

Grafico 2 Perfil de temperatura dentro de los secadores solares durante la operación de secado

La velocidad del aire en el secador solar que operó en convección forzada fue de $3 \mathrm{~m} / \mathrm{s}$, proporcionando un caudal de $5.3 \times 10^{-2} \mathrm{~m}^{3} / \mathrm{s}$, el cual se alimentó a temperatura del medio ambiente $\left(25 \pm 3{ }^{\circ} \mathrm{C}\right)$.

CARRILLO-CARRILLO, Manuela, CASTORENA-ALEMÁN, Juan Daniel, GARCÍA-JIMENÉZ, Fátima de los Ángeles y GARCÍAGONZÁLEZ, Juan Manuel. Deshidratación de plátano (Musa paradisiaca) por medio de radiación solar en un secador directo. Revista de Sistemas Experimentales. 2019 
De acuerdo a lo observado se pierde 1.72 $\pm 0.27 \mathrm{~g}_{\mathrm{agua}} /{ }^{\circ} \mathrm{C}$ en el deshidratado del plátano utilizando secadores solares directos.

De acuerdo a Bianchi et al (2011), mediante la deshidratación parcial o con aire caliente no es microbiológicamente estable, y además es más susceptible al deterioro químico, por lo cual se requiere un procesamiento posterior para incrementar la vida de anaquel (Bianchi, 2011).

La contribución con este estudio es que el deshidratado se realizó mediante aire a temperatura ambiente, suministrado a la caseta de secado solar directo, lo cual le permite conservar un color más atractivo del producto, así como, la calidad del producto.

\section{Conclusiones}

La mejor opción de secado en cuanto a economía es la natural ya que no requiere de algún aparato que le auxilie. Como se observó en los resultados fue muy poco la diferencia que tiene el porcentaje de humedad final en ambos regímenes de operación, por lo cual en este punto se puede decir que ambas son buenas y útiles para el secado, pero teniendo en cuenta el mercado con el que se trabaja en la actualidad se debe tomar en cuenta el color que se obtiene al final del secado y por este tema se tiene que la mejor operación es mediante convección forzada ya que preserva su color natural.

\section{Referencias}

Ángel Nolasco, S. A. (2015). "Desecado de la pulpa de plátano (Musa paradisiaca L.) utilizando un deshidratador solar con cristal transparente, bajo las condiciones climáticas de la comarca lagunera. Torreón, Coah.

Bianchi, M. G. (2011). DEHIDROCONGELACIÓN DE FRUTAS: ESTUDIO DE LOS PARÁMETROS DE CALIDAD. INVENIO 14 (26) , 117-132.

Carranza J., S. M. (2002). Cinética de secado de Musa paradisiaca L. "plátano" y Manihot esculenta Grantz "yuca". Revista Amazónica de Investigación. Vol. 2, No. 3, 15-25.
De la Vega, S. F. (2017). Diseño y construcción de un deshidratador de plátano mediante el aprovechamiento de energía solar pasiva para los laboratorios de la facultad ingeniería civil y mecánica. Ambato: Universidad Técnica de Ambato (tesis).

Dejo, F. T. (2018). Obtención y evaluación sensorial de galletas a diferentes concentraciones de harina de cáscara de plátano (Musa paradisiaca). Lambayeque, Perú: UNIVERSIDAD NACIONAL PEDRO RUIZ GALLO FACULTAD DE INGENIERIA QUÍMICA E INDUSTRIAS ALIMENTARIAS ESCUELA PROFESIONAL DE INGENIERÍA DE INDUSTRIAS ALIMENTARIAS.

Figueredo Rondón M.C., G. G. (2016). Evaluación del secado de la Musa Paradisiaca (plátano) utilizando el aparato de laboratorio SBAN. El Tigre, Anzoátegui, Venezuela: Universidad Politécnica Territorial José Antonio Anzoátegui .

Hernández, G. V. (2017). Secado de frutas y verduras con energía solar. Revista de Sistemas Experimentales, Vol. 4, No. 11., 22-33.

Minolta, K. (09 de 2014). Entendiendo El Espacio de Color CIE $L * A * B *$. Recuperado el 29 de 09 de 2019, de http://sensing.konicaminolta.com.mx/2014/09/e ntendiendo-el-espacio-de-color-cie-lab/

Paiva Purizaca, E. D. (2019). Colección, montaje e identificación de thrips de la mancha roja. Piura, Perú: UNIVERSIDAD NACIONAL DE PIURA, FACULTAD DE AGRONOMÍA, ESCUELA PROFESIONAL DE AGRONOMÍA .

Valero, G. T. (2018). La alimentación española características nutricionales de los principales alimentos de nuestra dieta. Madrid, España: Ministerio de Agricultura, Pesca y Alimentación Secretaría General Técnica Centro de Publicaciones. 